\title{
Verändern Medien die Sprachqualität?
}

\section{Das Wort im Stadium seiner technischen Reproduktion}

\section{von Eugen Biser}

Der Titel dieser Überlegungen enthält einen kaum verhüllten Hinweis auf den programmatischen Aufsatz „Das Kunstwerk im Zeitalter seiner technischen Reproduzierbarkeit", in welchem Walter Benjamin über den Bruch in der Geschichte des Kunstwerks nachsann, der durch den im Titel seiner Abhandlung angesprochenen Tatbestand eintrat. Zwar sei das Kunstwerk, meint Benjamin, „grundsätzlich immer reproduzierbar gewesen "1. Mit der Erfindung des Holzschnitts aber sei es erstmals "technisch reproduzierbar" geworden; und seit der Erfindung der Photographie habe sich der Ausstoß der Reproduktionen so sehr beschleunigt, „daß er mit dem Sprechen Schritt halten konnte". Inzwischen aber habe sich dieser Prozeß derart perfektioniert, daß eine Situation absehbar geworden sei, wie sie Paul Valéry mit den Worten kennzeichnete:

Wie Wasser, Gas und elektrischer Strom von weit her auf einen fast unmerklichen Handgriff hin in unsere Wohnungen kommen, um uns $z \mathfrak{u}$ bedienen, so werden wir mit Bildern oder mit Tonfolgen versehen werden, die sich, auf einen kleinen Griff, fast ein Zeichen einstellen und uns ebenso wieder verlassen. ${ }^{2}$

Deutlicher hätte das Fernsehen mit seiner elektronischen Steuerung kaum vorhergesagt werden können. Benjamin zieht aus dieser Prognose zwar keine so weittragenden Konsequenzen wie Freud in seinem berühmten Essay über "Das Unbehagen in der Kultur" (von 1930), die ihn geradezu von der Heraufkunft eines "Prothesen-Gottes“ reden lassen; doch spricht er von der tiefgreifenden Veränderung, die in den künstlerischen Verfahrensweisen dadurch eingetreten sei, daß das Kunstwerk nunmehr wie niemals zuvor zum Objekt wurde und als solches, vor allem in Form des Films, ,auf die Kunst in ihrer überkommenen Gestalt" zurückzuwirken begann.

Mit dieser signifikanten Ausdehnung der Einflußsphäre geht jedoch ein ungleich schwererer Verlust Hand in Hand. Die technische Reproduktion setzt an die Stelle des einmaligen Vorkommens des Kunstwerks "sein massenweises“. Es gewinnt eine zwar wirksame, im Grunde aber doch unechte Ubiquität; es kommt in aller Hand. So verliert es sein einmaliges Hier und Jetzt, grundsätzlicher noch gesagt, seine Echtheit. Seine geschichtliche Zeugenschaft gerät ins Wanken und mit ihr seine Autorität. Dem fügt Benjamin hinzu:

Man kann, was hier ausfällt, im Begriff der Aura zusammenfassen und sagen: was im Zeitalter der technischen Reproduzierbarkeit des Kunstwerks verkümmert, das ist seine Aura. ${ }^{3}$

\section{Die Verflachung des Wortes als Folge seiner elektronischen Reproduktion}

So weitet die technische Reproduktion den Horizont der Verfügbarkeit des Kunstwerks zwar ins Ungemessene; doch erleidet es gleichzeitig das, was Benjamin den "Verfall der Aura“ nennt." Damit trifft er eine Feststellung, die nicht nur von fun-

Prof. DDr. Eugen Biser leitet am Institut für Philosophie der Universität München das Seminar für christliche Weltanschauung und Religionsphilosophie. 
damentaler Bedeutung für das Verständnis der Kunst unter den Bedingungen der technischen Lebenswelt ist, sondern ebenso aufschlußreich für das Verständnis der im Dienst der Kommunikation stehenden Apparaturen. Im Grunde schreien Benjamins Gedanken schon seit ihrer Veröffentlichung im Jahre 1955 danach, in beiden Richtungen fortentwickelt zu werden. Denn was er zeigt, gilt nicht nur für den von ihm ins Auge gefaßten Fall eines reproduzierten Gemäldes, sondern unter der Voraussetzung, daß von ihm die Funktionsweise der technischen Reproduktionsverfahren zutreffend bestimmt wurde, mindestens ebensosehr auch für das technisch reproduzierte Wort. Sein Schicksal beim Durchgang durch die reproduzierenden Apparaturen soll im folgenden näher bedacht werden.

Was dem Wort bei seiner Wiedergabe durch ein elektronisches Medium im Extremfall widerfährt, kann am Beispiel seiner Umsetzung durch elektroakustische Geräte wie Harmonizer und Vocoder illustriert werden. Mit ihrer Hilfe ist es heute möglich, mit einer Wortfolge so zu verfahren, wie es der italienische Manierist Giuseppe Arcimboldo (von 1530-1573) bei der Erstellung seiner Porträt-Arrangements, den sogenannten „capricci“ tat, wenn er derartige Porträts aus Blumen, Laub oder Früchten zusammensetzte. ${ }^{5}$ Denn im Grenzfall kommt es dabei ebenso zu einer Demontage des Wortes, das lediglich seine „Formalgestalt" beibehält, während der Klang, anstatt von der menschlichen Stimme, von einem beliebig gewählten Geräusch oder Musikstück wie etwa von einem Bachschen Präludium - genommen wird. Der Vergleich mit dem "capricci“ drängt sich deswegen auf, weil auch dort der "Inhalt“ des Porträts nicht mehr vom menschlichen Gesicht, sondern von den nach seinen Strukturen arrangierten Gegenständen gebildet wird. Zwar entsteht bei entsprechender Kunstfertigkeit auch auf diese Weise echte Portraitähnlichkeit; doch kann nur noch in einem ganz entfernten Sinn davon die Rede sein, daß das Arrangement auch wirklich „der" mit ihm Dargestellte „sei“. Ebenso kann man eine aus Geräuschen oder Klängen montierte Wortfolge durchaus „verstehen“; doch wird man sich ernsthaft fragen müssen, was für ein "Verstehen " auf diesem Weg zustande kommt. Damit ist auch schon das Thema der folgenden Uberlegungen mit aller Deutlichkeit gestellt.

\section{Vorbedingungen, die Medien in ibrem Ausdruck zu versteben}

Wenn der Einstieg in die Erörterung gelingen soll, müssen zwei Vorbedingungen gegeben sein. Sie betreffen zum einen jenen Grad von Sensibilisierung, der erreicht sein muß, wenn der angesprochene Unterschied überhaupt wahrgenommen werden soll. Und sie betreffen zum anderen ein Sprachverständnis, das geeignet ist, in diese Differenz theoretisch hineinzuleuchten.

\subsection{Verflachung der Kommunikation - Versuch einer Sensibilisierung}

Was die Frage der Sensibilisierung anlangt, so ist diese keineswegs selbstverständlich. Vielen Zeitgenossen fällt kaum ein Unterschied auf, wenn sie die Schallplattenaufnahme einer Sinfonie hören und wenn ihnen diese Sinfonie, womöglich mit demselben Orchester und Dirigenten, im Konzertsaal begegnet. Beide Male glauben sie dasselbe gehört zu haben, obwohl Sergio Celibidache schon vor Jahren temperamentvoll darauf hinwies, daß von einer Identität nicht die Rede sein könne. ${ }^{*}$ Ähnlich ergeht es ihnen beim Vergleich eines öffentlichen Vortrags mit seiner Wiedergabe im Rundfunk. Tatsächlich ist jedoch die technische Reproduktion in beiden Fällen vom „Original“ grundverschieden. Und das nicht etwa nur hinsichtlich der ganz anderen akustischen Werte und der auch für den Laien hörbaren Einebnung der Klangdifferenz, also des 
Gefälles zwischen betonten und leise vorgetragenen Stellen, sondern hinsichtlich der Sache selbst. Schallplatte, Tonband und Rundfunk bieten zwar eine exakte „Klangkopie" des Originals, niemals aber ein Aquivalent von diesem selbst.

So deutlich dieser Unterschied für den ist, der ihn erst einmal wahrgenommen hat, so schwer fällt es gemeinhin, das Organ für seine Wahrnehmung zu entwickeln. Noch am ehesten gelingt das auf einem indirekten Weg, der von einem Wirkungsvergleich ausgeht. Dafür kann das deutsche Äquivalent zu der während der letzten Jahre in den Vereinigten Staaten entstandenen „elektronischen Kirche“ einen nützlichen Fingerzeig geben. Während sich die Kirchen zunehmend entleeren, ist - nahezu umgekehrt proportional dazu - ein ständiger Anstieg der Einschaltquoten bei religiösen Rundfunksendungen zu verzeichnen. Das Verhältnis ist so ausgeglichen, daß sich die Kirchenleitungen darüber nahezu beruhigen könnten, wenn da nicht ein signifikanter Unterschied bestünde. Denn während die gottesdienstliche Predigt, die den zentralen Vergleichspunkt bildet, ihrer ganzen Funktion nach zur Konsolidierung der Gemeinde beiträgt, ist das bei den - qualitativ oft höherstehenden - Rundfunkansprachen keineswegs der Fall. Sie führen eher zu dem entgegengesetzten Effekt einer zunehmenden Anonymität und Privatisierung des Religiösen. Auch in den Ausnahmefällen, in denen Hörer Kontakt zu dem jeweiligen Autor der Sendung suchen, verfolgt diese "R R̈̈ckmeldung" kaum einmal die Absicht, den Anschluß an eine Gemeinde, und wäre es auch nur seine fiktive „Hörergemeinde“, zu gewinnen.

\subsubsection{Information statt Kommunikation}

Daraus ergibt sich bereits ein wichtiger Rückschluß auf die spezifische Wirkung seines Wortes, das sich in der Regel inhaltlich kaum von dem einer Predigt unterscheidet. Es wirkt zwar informativ, da sich sonst die wachsenden Einschaltquoten nicht erklären ließen, gleichzeitig aber auch - und dies ganz unabhängig von der Intention seines Sprechers - isolierend. Zumindest geht ihm der konsolidierende Effekt, der sich zumindest intentional mit dem lebendigen Predigtwort verbindet, auf eine nicht zu übersehende Weise ab. Damit aber drängt sich auch schon der Gedanke an eine medienbedingte Transformation der Sprache so nachdrücklich auf, daß er nicht mehr von der Hand gewiesen werden kann.

Eine Episode der Apostelgeschichte kann verdeutlichen, daß es sich dabei um eine spezifische Wirkung des Mediums handelt, durch welche die wichtigste Wirkung der mit dem Martyrium des Stephanus einsetzenden Verfolgung der jungen Kirche, ihre beginnende Missionstätigkeit, demonstriert werden soll. Es ist der Bericht von der Bekehrung des äthiopischen Kämmerers, der sich während einer Pilgerreise nach Jerusalem das Buch des Propheten Jesaja - vermutlich in Gestalt einer der im Wüstenkloster Qumran angefertigten Schriftenrollen - erstanden hatte, und auf seiner Rückreise, auf seinem Wagen sitzend, in dem prophetischen Buch liest und dabei auf eine Textstelle stößt - das Lied vom Gottesknecht (Jes 53) - die ihn zwar seltsam anrührt, ihm jedoch unverständlich bleibt (Apg 8,26-40). Von einem Engel angewiesen, tritt ihm dann aber als hilfreicher Interpret der Diakon Philippus in den Weg, der ihm die dunkle Stelle so wirkungsvoll erklärt, daß er sich von ihm an Ort und Stelle taufen läßt:

Da begann Philippus zu reden, und ausgehend von dem Schriftwort verkündete er ihm das Evangelium von Jesus. Als sie nun weiterzogen und an eine Wasserstelle kamen, sagte der Kämmerer: Hier ist Wasser; was steht meiner Taufe noch im Weg? Da ließ er den Wagen halten und beide, Philippus und der Kämmerer, stiegen in das Wasser hinab, und er taufte ihn $(8,35-38)$. 


\subsubsection{Vom toten Buchstaben zum „lebenspendenden Geist ${ }^{\text {“ }}$ durch unmittelbar personale Vermittlung}

Obwohl mit dem schriftlichen Text bereits alles gesagt ist, klärt sich sein Sinn doch vollends erst im Dialog mit dem Interpreten. Jetzt erst verwandelt er sich, mit einer paulinischen Differenz ausgedrückt, vom „toten Buchstaben“ in den „lebenspendenden

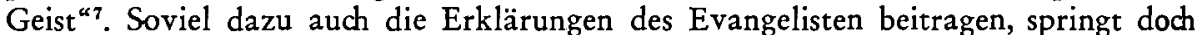
die von der Erzählung zumindest angedeutete Tatsache in die Augen, daß es erst dem mündlichen Wort gegeben ist, den vom Text Betroffenen zu praktischen Konsequenzen aus der gewonnenen Einsicht zu bewegen. Der Sinn mußte zunächst aus der Form, wie er im Medium enthalten war, „erhoben" werden, um zu seiner umgestaltenden Wirksamkeit zu gelangen. Diese Freisetzung erfolgte im lebendig gesprochenen Wort.

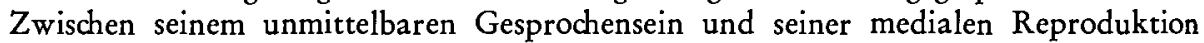
besteht somit - trotz inhaltlicher Invarianz - ein Unterschied von jener Größenordnung, wie er zwischen Theorie und Praxis vorliegt.

\subsection{Ein umfassendes Sprachverständnis}

Wenn diese Differenz nicht nur dunkel gefühlt und staunend registriert werden soll, muß sie durch ein Sprachverständnis unterbaut werden, das den aufgezeigten Tatbeständen gerecht wird. Das aber vermag keine Sprachtheorie zu leisten, die sich im Interesse maximaler Exaktheit von vornherein auf die quantifizierbaren Daten der Sprechhandlung und des Sprachgeschehens beschränkt. Auch das instrumentelle Sprachverständnis der analytischen Sprachanalyse, das inhaltsbezogene der Sprachinhaltsforschung und das ausdrudksbezogene des Strukturalismus greifen in dieser Frage zu kurz. Selbst mit der Einbeziehung der performativen Sprachqualitäten im Sinn des Ansatzes von John L. Austin und seiner Frage „How to do things with words?" ist es nicht getan. Erforderlich ist vielmehr ein Sprachverständnis, das bei aller Betonung der instrumentellen Leistung der Sprache zugleich auf ihre Konsubstantialität mit dem Menschsein achtet. Denn ebenso richtig wie die Feststellung, daß der Mensch über seine Sprache verfügt und sie auf unterschiedliche Weise zu gebrauchen weiß, ist die andere, wonach er seine Sprache nicht hat, sondern ist. Das machen vor allem die Unterschiede in der Sprachverwendung deutlich. Wie insbesondere die Erfahrung mit der politischen Rede lehrt, kann Sprache auf eine höchst verschiedenartige Weise eingesetzt werden: als Demonstrationsobjekt ebenso wie als Mittel der Positionsbestimmung und als Instrument der Propaganda oder, im gegenteiligen Fall, der Selbstverheimlichung und Tarnung. Indessen genügt es, diese unterschiedlichen Formen der Sprachverwendung aufzuführen, um sie auch schon als ausgesprochene Fehlformen zu erkennen. Das aber lenkt auch schon den Blick zurück auf jene elementare Sprechintention, die mit der Menschlichkeit der Sprache gegeben ist. Doch worin besteht sie?

\subsubsection{Sprache als Mitrel zur Uberwindung von Einsamkeit}

Wenn man davon ausgeht, daß mit jedem Sprechakt - auch dem noch so sachlich gemeinten - eine unwillkürliche Selbstanzeige verbunden ist, kann die Antwort nur lauten: in dem mit jedem Wort gegebenen Selbsterweis liegt die Absicht des Sprechenden. Wie das eklatante Mißverhältnis zwischen der Menge der in jedem Gespräch verwendeten Sprachzeichen und der Menge der mit ihnen tatsächlich gegebenen Informationen zeigt, reden wir nicht deswegen, weil wir, wie die landläufige Ansicht meint, einander etwas zu sagen haben, sondern weil wir einander brauchen. Dabei kommt, motivationsanalytisch gesehen, der Informationswille oft nur nach Art eines Vorwands ins Spiel. Zwar lassen wir uns vordergründig bei unserem Reden von Mittei- 
lungswille und Neugier leiten, doch folgen wir dabei letztlich dem Wesenszug zur Uberwindung der Einsamkeit. Sprache ist, so gesehen, der symbolhafte Exzeß des Menschseins, durch den wir in Akten gegenseitiger Anerkenntnis und Bestätigung einander immer neu zu verstehen geben, daß für uns ein sinnerfülltes Dasein nur in der gegenseitigen Verbundenheit möglich ist. So bekunden wir dem Gesprächspartner in jedem wesentlich gesprochenen Wort, daß es uns auf ihn ankommt, daß uns an ihm gelegen ist und daß wir, zumindest für die Dauer des Gesprächs, eine zuständliche Verbindung mit ihm erstreben. Das steht im Hintergrund der großen Wiederentdeckung der Sprache, die noch vor den Initiatoren der analytischen Sprachphilosophie durch die Denker des dialogischen Prinzips vollzogen wurde. Vor allem aber spricht es aus der programmatischen Gleichsetzung von Wort und Liebe im Werk Ferdinand Ebners, der deshalb als der entscheidende Pionier des integralen, und das heißt anthropologisch fundierten Sprachverständnisses zu gelten hat. ${ }^{8}$ Denn es kam einem geistigen Durchbruch gleich, wenn Ebner die programmatischen Worte schreiben konnte:

Es gibt zwei Tatsachen, nicht mehr, des geistigen Lebens, zwei Tatsachen nämlich, die sich zwischen dem Ich und dem Du zutragen: Das Wort und die Liebe. In ihnen liegt die Erlösung des Menschen, die Befreiung seines Ichs aus seiner Selbstabschließung.9

\subsubsection{Durch Medienkonsum in die Einsamkeit}

Wenn es zutrifft, daß beim elektronisch übermittelten Wort die informative Komponente derart überwiegt, daß es, soziologisch gesehen, eher in die Vereinsamung und Privatisierung treibt, wird jetzt, vor diesem theoretischen Hintergrund, der von den Medien in Gang gesetzte Transformationsprozeß erst voll ersichtlich. Auch bei voller inhaltlicher Invarianz ist die aktuell gesprochene Rede deutlich verschieden von dem, was die elektronische Ausstrahlung von ihr wiedergibt. Umgekehrt heißt das dann aber auch, daß die auf ein instrumentelles Sprachverständnis eingeschränkten Theorien in einer ebenso bedenkenswerten wie bedenklichen Ubereinkunft mit dem stehen, was der Sprache im Zeitalter ihrer technischen Reproduktion widerfährt. Um so mehr ist Anlaß gegeben, dieser Transformation genauer nachzugehen.

\section{Die Reduktion: technische Apparaturen jenseits von Einsamkeit und Gemeinschaft}

Den Versuch, dem Minderungseffekt auf die Spur zu kommen, den eine elektronisch vermittelte Aussage erleidet, steht heute, anders als in den Tagen Benjamins, eine erhebliche Schwierigkeit entgegen. Denn inzwischen wurde fraglos das Fernsehen zum dominierenden Medium. Von ihm aber gilt wie von keinem andern der programmatische Satz Marshall McLuhans, "the medium is the message - das Medium ist die Botschaft ${ }^{{ }^{110}}$. Denn im Fall des Fernsehens setzt sich das Medium derart gegen den jeweils vermittelten Inhalt durch, daß die von ihm ausgehende Faszination auch bei sehr unterschiedlichen Inhalten annähernd gleichbleibt. Von dieser Uberlagerung durch die „Eigenmächtigkeit“ des Mediums muß aber abgesehen werden, wenn die an der Aussage auftretende Veränderung in den Blick gebracht werden soll. Von ihr wurde bisher immerhin soviel deutlich, daß von einem Subtraktionseffekt gesprochen werden kann. Das elektronisch vermittelte Wort verliert eindeutig an Dignität und Profil. Es wirkt gegenüber dem lebendig gesprochenen eigentümlich verflacht, so daß man versucht ist, den von Herbert Marcuse geprägten Ausdruck von der "Eindimensionalität ${ }^{\text {“ }}$ zur Verdeutlichung heranzuziehen. ${ }^{11}$ Tatsächlich erleidet die elektronisch vermittelte 
Sprache einen signifikanten Profilverlust, der insbesondere die für die Selbstdarstellung und Mitmenschlichkeit ihres Sprechers bedeutsamen Qualitäten betrifft. Das Wort des Radiosprechers wirkt, verglichen mit dem des konkreten Gesprächspartners, eigentümlich hintangehalten und abstrakt, ganz so, als sei das übermittelnde Medium für eine menschliche Aussage nur partiell durchlässig.

Es ist schwer zu entscheiden, ob es mit diesem Dignitätsverlust zusammenhängt odet ob, zusammen mit ihm, eine gegenläufige Tendenz am Werk ist; auf jeden Fall aber tritt das informative Element beim elektronisch vermittelten Wort deutlicher als beim dialogisch gesprochenen hervor. Es ist tatsächlich so, daß im selben Maß, wie die $\mathrm{Di}$ gnität des Wortes verblaßt, sein Informationswert in den Vordergrund tritt. Bei störungsfreier Ubermittlung ist das Wort des Rundfunksprechers leichter zu verstehen als das konkret gesprochene, doch so, daß es gleichzeitig weniger „anspricht“, bewegt und ergreift.

Dem halte man nicht die Wirkung der Hitler- und Goebbels-Reden im Dritten Reich entgegen! Denn diese wirkten, soweit sie durch Großlautsprecher zu Massenversammlungen übertragen wurden, eher einschüchternd und furchterregend als faszinierend. Bezeichnend dafür ist die „Sportpalastrede“, in welcher Goebbels zum totalen Krieg aufrief. Denn im Unterschied zu seinen aktuellen Hörern, die ihm frenetisch zujubelten, war den Rundfunkhörern seiner Rede diese Reaktion ebenso unheimlich wie unverständlich. Auf sie übertrug sich die offensichtliche Faszination nicht oder höchstens in Form eines angsterregenden Schocks. Insofern bestätigen sogar diese extremen Beispiele einer totalitären Demagogie den aufgezeigten Befund. Sie zeigen überdies, daß der registrierte Subtraktionseffekt durch kein zusätzliches Element aufgewogen werden kann. Weder erreichte die exaltierte Rhetorik dieser Propagandareden das Urteils- und Entscheidungszentrum ihrer Hörer, noch wurden diese durch die gewaltige Phonstärke der Ubermittlung zu dem gebracht, wozu das wesentlich gesprochene Wort auch ohne jeden Stimmaufwand bewegt. So bleibt es bei der Feststellung, daß die elektronische Ubermittlung dem Wort das entzieht, wodurch es nicht nur Wissen, sondern Gewißheit stiftet. Und das ist primär die Gewißheit, daß der Redende für den Angesprochenen da ist und daß es ihm darauf ankommt, mit ihm in eine Gesprächsgemeinschaft, womöglich sogar in eine Aktions- und Lebensgemeinschaft zu treten. Vergegenwärtigt man sich überdies, daß der Informationsgehalt der Aussage gleichzeitig eine Verstärkung erfährt, so wird auch schon die Richtung deutlich, in welche die Sprachentwicklung durch die elektronischen Medien gewiesen wird. Es ist die Richtung, die zum Zielpunkt der Computersprache führt. Und wiederum wird eine eigentümliche Konvergenz der die Sprachwelt beherrschenden Faktoren und Tendenzen sichtbar. War es im ersten Fall die Übereinkunft von analytischer Sprachtheorie und elektronischen Medien, so handelt es sich jetzt, im zweiten Fall, um den Zusammenhang, in welchem diese mit der Entstehung einer formalisierten Sprache stehen. Nichts, so scheint es, kommt in diesem Bereich von ungefähr.

Die von dem Schlüsselsatz, der das Medium mit der Botschaft gleichsetzt, bekundete Beobachtung ist dazu angetan, dem verstärkten Informationswert der elektronisch vermittelten Aussage eine weitere Deutung zu geben. Voraussetzung dessen ist allerdings, daß man sich darauf einigen könnte, die Binnenstruktur der modernen Medien als "informatorisch“ zu bezeichnen. Das aber wird um so leichter möglich sein, als offenkundig am Anfang der Entwicklung die Erfindung der drahtlosen Telegraphie steht, die alles übrige, die Entstehung des Telefons, des Rundfunks und des Fernsehens, nach sich zog. Daß diesem eine so hohe Suggestivität eignet, daß sich für die Beschreibung der Fernsehgewohnheiten sogar Begriffe aus der Drogenpathologie ein- 
bürgerten - man spricht geradezu von „Fernsehentzug“ -, hängt zweifellos mit der Verkoppelung von visuellen und optischen Elementen zusammen, in der bereits Benjamin den Wendepunkt der Entwicklung erkannte. Von der bisherigen Analyse des Vorgangs wurde die Frage, ob dabei quasi-religiöse Elemente ins Spiel kommen, durchaus bejaht. In diesem Zusammenhang kam nicht nur die Rede von der mythenbildenden Rolle des Fernsehens auf; vielmehr sprach Harvey Cox, der durch das Buch „Stadt ohne Gott", das besser mit dem Originaltitel "The Secular City" (von 1965) zitiert werden sollte, berühmt gewordene Vertreter der Radikalen Theologie, geradezu von der "elektronischen Ikone", die durch das Fernsehen in die modernen Wohnungen eingezogen sei. ${ }^{12}$ Ungeachtet der Anspielung auf die Sakralwelt kommt das eher einer Bestätigung als einer Widerlegung der These von der - auch beim Fernsehen zu vermutenden - Informationsstruktur gleich. Denn mit den Begriffen „Mythos“ und "Ikone" soll im Grunde nur verdeutlicht werden, daß das Fernsehen zu ritualisierten Formen der Verknüpfung von Wort und Bild tendiert. Das aber liegt ganz auf der Linie der Tatsache, daß auch dieses Medium von seiner ganzen Struktur her der reinen Informationsvermittlung dient.

Bezieht man das auf die Frage nach der vom Wort erlittenen Transformation zurück, so könnte man diese nun auch dahin bestimmen, daß dem elektronisch vermittelten Wort die Eigenstruktur des Mediums aufgeprägt werde. Damit würde definitiv geklärt, warum es gerade der Informationswert ist, der nunmehr, in der elektronischen Ubermittlung, verstärkt zum Vorschein kommt. Von Ferne stellt sich damit nochmals die Erinnerung an die manieristischen Kompositionen Arcimboldos und die durch Harmonizer und Vocoder erzeugten Wortmontagen ein. Auch wenn aus dem Fernsehgerät eine ganz menschlich klingende Stimme ertönt, ist ihr Klang doch durch die Magie der Apparatur verwandelt, so daß er gleichzeitig durch ihren „Eigenton “ geprägt erscheint.

Sofern man dies zugibt, ist auch schon ein Erklärungsgrund dafür gefunden, daß dem elektronisch übermittelten Wort - bei aller Reproduktionstreue - die Sprachqualitäten abgehen, die der sprachlichen Selbstdarstellung und Solidarisierung dienen. Denn die technische Apparatur steht jenseits von Einsamkeit und Gemeinschaft. Sie kennt weder die Not dessen, der über die Grenzen seiner Individualität hinausstrebt, noch das freudige Erschrecken des andern, der zum Adressaten einer sprachlichen Zusage wurde, und schon gar nichts weiß sie von dem Glück, das beide miteinander verbindet, wenn der Brückenschlag gelang.

\section{Die Auswirkungen}

Um die Auswirkungen zu ermessen, genügt es, sich den Zustand als Regelfall vorzustellen, der in vielen Familien längst schon Abend für Abend eingetreten ist: die Verdrängung des dialogischen Gesprächs durch die aus dem Lautsprecher erklingenden Stimmen. Es ergäbe sich ein höchst widersprüchlicher Zustand, in dem ein elementares Defizit durch einen Verstärkereffekt überdeckt wird. Das Defizit beträfe die personalisierenden und konsolidierenden Sprachqualitäten, der Verstärkereffekt bezöge sich dagegen auf den Informationswert. Um dabei einzusetzen, so wäre an erster Stelle ein signifikantes Anwachsen des Informationsstandards zu verzeichnen. Angesichts der Bevorzugung von Unterhaltungssendungen, die auch bei geringster Qualität auf hohe Einschaltquoten spekulieren können, leuchtet das nicht ohne weiteres ein. Was aber haften bleibt, sind auf die Dauer doch die als "interessant“ empfundenen Sendungen; 
und in dieser Hinsicht stehen eindeutig die Nachrichtensendungen an der Spitze. Somit bleibt es auch angesichts dieser scheinbaren Ausnahme bei der Feststellung, daß mit dem Abbau der menschlichen und mitmenschlichen Implikationen eine Verstärkung des Informationswerts einhergeht.

\subsection{Probleme religiösen Redens mittels elektronischer Medien}

Auf das Glaubensbewußtsein bezogen, ergibt sich damit eine in glaubenstheoretischer Hinsicht seltsame Umschichtung. Während es dem Zug der modernen Theologie entspricht, daß im Unterschied zur neuscholastischen Akzentuierung des Glaubens der subjektive Anteil, also die "fides qua “ stärker hervorgehoben wird, drängt der Medieneinfluß das Glaubensbewußtsein wieder eher auf die alte Position zurück, bei welcher der Glaubensinhalt, also die "fides quae“, im Vordergrund stand. Von dieser Wirkung her müßte man somit geradezu von einer "Antiquiertheit“ der modernen Medien und ihrer Auswirkungen sprechen. ${ }^{13}$ Die Tatsache selbst läßt sich indessen kaum bezweifeln. Denn schon eine vergleichsweise geringfügige Medienerfahrung lehrt, daß sich paränetische und erbauliche Inhalte nur schlecht übertragen lassen. Von Sendungen einer betont erbaulichen Tendenz fühlen sich die Hörer eher abgestoßen, Imperativen gegenüber reagieren sie geradezu allergisch. Um so lieber werden Rundfunk und Fernsehen als Quellen einer "gehobenen Information“ genutzt. Das hängt zweifellos damit zusammen, daß das elektronische Medium informativen Inhalten gegenüber besonders konform ist. Sachaussagen kommen am besten an. Diese schlichten Erfahrungsdaten drängen geradezu darauf, „taktisch“ genutzt zu werden. Wie könnte das geschehen?

Ein erster Schritt wäre bereits mit der Verabschiedung der immer noch vertretenen Auffassung getan, daß sich eine für Rundfunk oder Fernsehen bestimmte Sendung möglichst eng am Modell eines Wortgottesdienstes auszurichten habe. Was dann zustande kommt, ist bekanntlich eher ein Gespenst als die wirkliche Wiedergabe eines derartigen Gottesdienstes. Nicht nur, daß von seiner "Weihe“ und Suggestivität kaum etwas zu rekonstruieren ist; auch seine einstimmende Funktion erweckt den Eindruck, auf unerklärliche Weise „suspendiert" zu sein. Daraus ergeben sich die positiven Entscheidungen fast von selbst. An die Stelle des Versuchs, dem Medium die ihm fremde Form gottesdienstlicher Spontaneität aufzudrängen, muß die Bereitschaft treten, die Aussage medienkonform zu gestalten. Bevor davon jedoch eingehender die Rede sein kann, muß dem Transformationsprozeß noch genauer nachgegangen werden.

\subsection{Ritualisierung übermittelter Nachrichten}

So schwer die Verlagerung der Gesamtaussage auf ihren Informationswert hin gerade für die religöse Verkündigung zu Buch schlägt, ist sie von zwei weiteren Tendenzen doch nicht weniger schwer betroffen. Die eine betrifft den Effekt, mit dem die Medien das durch sie verursachte "Erfahrungsdefizit" kompensieren; die andere besteht in der „Ritualisierung“ der durch sie übermittelten Nachrichten. Um dabei einzusetzen, so muß dieser im Grunde schon von Benjamin beobachtete Effekt im Zusammenhang mit der Informationsverstärkung gesehen und als deren gegenläufige Minderung in Anschlag gebracht werden. Auch in dieser Hinsicht ist die Funktionsweise der Medien dialektisch. Wie ihre Modernität, von der religiösen Wirkung her gesehen, zugleich anachronistisch wirkt, so treten sie auch in qualitativer Hinsicht ihren Wirkungen selbst in den Weg. Was insbesondere den durch sie verstärkten Informationswert anlangt, so wird er nachhaltig dadurch gemindert, daß die Aussage meist in einer schematisierten Form erfolgt. Dabei kommen ebensosehr Rücksichten auf ihre Verständ- 
lichkeit wie auf die vermutete Sinnerwartung des Publikums ins Spiel. Deutlicher noch als im Rundfunk ist das in Film und Fernsehen der Fall, wo sich die "Logik" der Abfolge unverkennbar am Publikumsgeschmack bemißt und wo selbst dann, wenn das Unerwartete geschieht, es im Blick auf eine Erwartungshaltung geboten wird. Diesem Prozeß muß um so größere Aufmerksamkeit gewidmet werden, als er sich nach Art einer gegenseitigen Eskalation vollzieht. Indem die Medien ihre Nachrichten im Sinn einer vermuteten Publikumserwartung ritualisieren, führen sie entweder beim Publikum zu einer entsprechenden Erwartungshaltung, oder bestätigen sie diese, sofern sie tatsächlich schon vorliegt. Daß damit eine ständige Qualitätsminderung verbunden ist, liegt auf der Hand. Denn im Zug einer durchaus absehbaren Entwicklung kommt es hierdurch zu einem zweiten "Ausfilterungseffekt", der sich jetzt auf die Informationsgehalte selbst bezieht. Betraf der erste, sprachtheoretisch gesehen, die persuasiven, evidenz- und empirievermittelnden sowie die solidarisierenden Sprachfunktionen, so verhindert es dieser zweite zunehmend, daß Informationen „höherer Ordnung " geboten werden können, wobei sich diese sowohl auf den mit ihnen verbundenen Anspruch als auch auf ihren Komplexitätsgrad bezieht. Was ankommt und deshalb allein auch noch gefragt ist, sind möglichst einfache, der Ordnung des Faktenwissens entnommene und im Sinne der Leichtverständlichkeit organisierte Informationen. Das aber ist eine Entwicklung, die sich insbesondere auf den Bereich der Glaubensbildung nur verhängnisvoll auswirken kann.

\subsection{Medienkonsum als Sucht}

Zur dialektischen Wirkungsweise der Medien gehört aber auch, daß sie das mit ihnen gegebene Empiriedefizit kompensieren. Sowenig sie sich dazu eignen, Imperative geltend $\mathrm{zu}$ machen oder Erbauung und Trost $\mathrm{zu}$ vermitteln, und sowenig durch sie ein wirklicher Dialog zustande kommt, lassen sie doch die durch die spontane Sprachverwendung geweckten Erwartungen nicht leer ausgehen. Zwar vermitteln sie weder Ergriffenheit noch Trost, dafür aber das Gefühl eines diffusen „Gehobenseins", das sich im Extremfall zu einer narkotisierenden Wirkung verstärkt. Auch kommt es durch sie - selbst beim Telefongespräch — zu keiner echten Dialogsituation, wohl aber zur Suggestion eines Einverständnisses, die über das tatsächliche Defizit hinwegtäuscht. Im Gegenzug zu dem mit ihnen eintretenden Verlust an Primärerfahrungen vermitteln sie, mit Arnold Geblen gesprochen, „Erfahrungen zweiter Hand“"14.

Abgesehen davon, daß der Mensch in der industrialisierten Welt längst schon so sehr an Ersatzstoffe gewöhnt ist, daß er die durch sie vertretenen Naturprodukte weder vermißt noch gegen sie eintauschen möchte, kommt der Suggestivität dieser Erfahrungen zweiter Hand ein Dreifaches zugute: die Menge, in der sie geboten werden, die Leichtigkeit, mit der sie zu gewinnen sind, und die Präzision ihrer Ubermittlung. Die Präsentation eines Gemäldes im Fernsehen übertrifft, ihre technische Perfektion einmal vorausgesetzt, alles, was das Auge des Museumsbesuchers an Farbwerten und Konturen wahrzunehmen vermag. Genauso verhält es sich mit den Eindrücken, die von der Fernsehübertragung einer Papstreise vermittelt werden. Während hier die tatsächlichen Teilnehmer einer Veranstaltung selbst einen bescheidenen Randplatz mit stundenlangem Warten erkaufen müssen, wird dem Fernsehkonsumenten die Reproduktion des ganzen Geschehens ohne die mindeste Anstrengung „f rei Haus“ geliefert. Damit tritt auch schon ein erster Wesenszug in dieser "reproduktiven Sekundärerfahrung“ zutage: sie nimmt dem Rezipienten die Anstrengung ab, die mit der Primärerfahrung in der Regel verbunden ist. Noch gravierender ist ein zweites Moment: der Erfahrungshorizont weitet sich ins Grenzenlose. Die moderne Nachrichtentechnik ver- 
mittelt ebenso Einblicke in den Feinbau der Materie wie in die Struktur der Saturnringe, von den Vorkommnissen und Gegebenheiten exotischer Gebiete auf dieser Erde ganz zu schweigen. Und sie vermittelt dies buchstäblich mit Lichtgeschwindigkeit. Niemand braucht die Strapazen einer Reise auf sich zu nehmen, um sich einen Eindruck von den Skulpturen auf den Osterinseln oder von philippinischen Heilpraktiken $\mathrm{zu}$ verschaffen.

Nur zu leicht wird durch diese Fülle, Anschaulichkeit und Exaktheit das Defizit verschleiert, das mit Erfahrungen aus zweiter Hand einhergeht. Ihnen fehlt das Moment des Spontanen, den Rezipienten unmittelbar Betreffenden. Was sie ansprechen, ist zwar seine Aufmerksamkeit und Neugier, nicht jedoch er selbst in seiner Personmitte. So schaffen sie, wie man in Abwandlung eines Pascalschen Gedankens sagen könnte, eine "zweite Natur“, durch die der Mensch dazu gebracht wird, nicht nur von Reproduktionen, sondern geradezu als eine Reproduktion seiner selbst zu leben. Indem sie ihn bereichern, halten sie ihn zugleich von sich selber ab. Sie versetzen ihn in eine Art Sekundärform seiner selbst, die ihm das Gefühl einer umfänglichen Entlastung vermittelt, gleichzeitig aber auch die Kräfte seiner spontanen Lebensgestaltung untergräbt und schwächt. Daß er im Maß dieser Schwächung zugleich manipulierbar wird, versteht sich von selbst. Deutlichstes Symptom dessen ist die ihm durch die Medien aufgezwungene Konsumhaltung, die sich bis zur Süchtigkeit steigern kann. Nicht umsonst stehen die Medien für kritische Beobachter in der Nähe der Drogenszene, weil sie Berauschungs- und Entzugserscheinungen wahrzunehmen glauben, wie sie nur bei dieser auftreten. $\mathrm{Ob}$ damit allerdings, und wäre es auch nur im Blick auf die zukünftige Entwicklung, zu einem anthropologischen Pessimismus Anlaß gegeben ist, bleibe dahingestellt. Zwar ist jetzt schon abzusehen, daß der Medienkonsum aufgrund der bereits eingeleiteten Entwicklungen in einer noch kaum vorstellbaren Weise wächst; doch ist es keineswegs eine ausgemachte Tatsache, daß sich damit auch der Grad der menschlichen Entfremdung und Manipulierbarkeit steigert. Vielmehr könnte auch der von Siegmund Freud vorausgesehene Fall eintreten, daß schon der Mensch der näheren Zukunft mit den Medien "verwächst“, oder daß er sie zumindest nach Art von Prothesen eines gesteigerten, wenn auch keineswegs vergöttlichten Selbstseins nutzen lernt. ${ }^{15}$ Das aber heißt zugleich, daß mit dieser Entwicklung nicht notwendig eine religiöse Krise einhergeht, da sie zwar zu einem progressiven Verlust von Primärerfahrungen, nicht jedoch auch zu einer unausweichlichen Denaturierung des Menschen führt.

\section{Die Reaktion}

Wenn man von Entwicklungen nicht überrollt werden will, muß man auf sie reagieren. So auch hier. Dann aber lautet die Zentralfrage: wie kann im Zeitalter der technischen Promulgation des Wortes - besonders auch im religiösen Sprachraum - noch im Vollsinn des Wortes „menschlich“ gesprochen werden? Im Blick auf den aufgewiesenen Subtraktionseffekt ist das nahezu gleichbedeutend mit der Frage, wie angesichts der technisch bedingten "Sprachverschiebung“, um diesen an das Phänomen der "Rotverschiebung“ im Spektrum ferner Spiralnebel angelehnten Ausdruck zu gebrauchen, die Effizienz des Wortes gewahrt werden kann. Ihrer ganzen Tendenz nach zielt diese Frage auf eine zweifache Antwort: auf eine erste, die das Problem zunächst einmal theoretisch aufzuarbeiten sucht, und auf eine zweite, die daraus praktische oder doch wenigstens praxisorientierte Folgerungen zieht. 
Was erarbeitet werden muß, ist zunächst und vor allem eine möglichst umfassende Theorie der medienbedingten Transformation der Sprache. Vom Umfang der damit gestellten Aufgabe gewinnt man dann ein Bild, wenn man sich vergegenwärtigt, daß sich im Umgang mit den elektronischen Medien der Sinn für medienspezifische Sprachveränderung insgesamt schärfte, so daß das Problem nicht nur dort auftaucht, wo es im Zug moderner Medienerfahrung entdeckt wurde, sondern auch dort, wo es längst schon bestand, wo er mit Ausnahme von wenigen besonders sensiblen Geistern so gut wie unbeachtet blieb: im Bereich der Schriftkultur. ${ }^{16}$ Doch hätte sich wenigstens die Christenheit schon durch den zu einem angemessenen Problembewußtsein führen lassen können, der das Medium Schrift als erster in den Dienst der Verkündigung stellte und dabei nicht nur die mit ihm eröffneten Möglichkeiten, sondern auch die ihm gezogenen Grenzen vor Augen hatte: Paulus. ${ }^{17}$

\subsection{Grenzen der medialen Verkündigung anhand der christlichen Schriftkultur}

Paulus setzte das Medium des apostolischen Briefs mit großer Zielsicherheit ein, wenn, wie im Fall der im Glauben verunsicherten Gemeinden von Galatien, rasches Eingreifen geboten, ein persönlicher Besuch jedoch durch die Umstände unmöglich war; doch begnügt er sich im Bewußtsein der Leistungskraft des von ihm verwendeten Mediums damit, seine Adressaten daran zu erinnern, daß er ihnen in seinem Predigtwort den gekreuzigten Christus mit der Suggestivität einer Ikone "vor Augen gezeichnet " habe (Gal 3, 1). Gleichzeitig wünscht er sich, „mit anderer Stimme“, und das besagt: mit der Uberredungskraft des mündlichen Wortes, zu ihnen reden zu können $(4,20)$, weil er durch ihr Verhalten ganz ratlos geworden sei. Und der Apostel verweist nicht nur auf die Grenzen des von ihm eingesetzten Mediums; er entwirft auch schon die Grundzüge einer medienkritischen Sprachtheorie, wenn er den "lebendigen Geist" vom

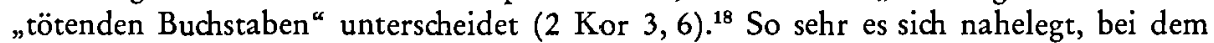
todbringenden "Gramma" mit Erhard Kamlab an das jüdische Gesetzbuch zu denken, zielt der von Paulus verwendete Ausdruck doch zweifellos auch auf das, was jede Schrift mit dem "Gesetz" gemeinsam hat. Denn alle Schrift tendiert zur Vorschrift, sofern sie das in ihr Niedergelegte in die Form eines "So-und-nicht-anders" bringt. So geht es ihr schon von ihrer Entstehungsgeschichte her nach, da sich die große Mehrzahl der ältesten Schriftdokumente auf Gesetzestexte bezieht. Doch unabhängig davon vollzieht sich mit jedem Akt der Verschriftung eine Festlegung des frei gesprochenen, lebendigen Wortes, so daß es sich fast von selbst ergab, daß die Kirchenväter in diesem Vorgang eine Analogie zur Menschwerdung des ewigen Wortes erblidkten. ${ }^{19}$ Diese restriktive Tendenz muß schon Paulus deutlich geworden sein, als er das $\mathrm{Me}$ dium Schrift in den Dienst seiner Verkündigung stellte. Bisweilen muß es ihm geradezu vorgekommen sein, als habe er damit die Spontaneität des dialogisch gesprochenen Wortes in die Enge des Buchstabens "begraben“.

Von den großen Erneuerern des Paulinismus widmete Augustinus immerhin ein ganzes Werk dem Thema "Geist und Buchstabe" (von 412), mit dem er sich allerdings, wie er abschließend zu verstehen gibt, hauptsächlich gegen ein am Literalsinn klebendes Schriftverständnis und eine Gesetzeserfüllung aus dem Geist der Furcht wendet. ${ }^{20}$ Um so sensibler ging Luther auf die von Paulus ausgearbeitete Differenz ein, indem er unterstrich, daß mit "Evangelium eigentlich nicht Schrift, sondern mündliches Wort ${ }^{\text {“ }}$ gemeint sei, so daß es einem "großen Abbruch“ und einem "Gebrechen des Geistes“ gleichkomme, daß, von der „Not erzwungen“, überhaupt Bücher geschrieben werden mußten. ${ }^{21}$ Damit greift er einen Gedanken auf, der sich schon bei einem der frühesten Informanden über die Entstehungsgeschichte des Christentums, bei Papias von Hierapolis, findet, wenn er bemerkt: 
Ich war immer der Meinung, daß das, was von Büchern stammt, für mich keinen ebenso großen Wert haben könne, wie das, was von lebendiger und bleibender Stimme kommt. ${ }^{22}$

Doch wichtiger als diese Reminiszenz ist die Fortführung der von Luther geübten Medienkritik bei Lessing, der sich wiederholt über die Abkünftigkeit der biblischen Schriften äußert und dem zur Schriftreligion erstarrten Christentum in seinem Essay „Uber den Beweis des Geistes und der Kraft" (von 1777) geradezu vorwirft, zu einer Reproduktion seiner selbst herabgesunken zu sein. Niemand machte sich das nachdrücklicher zu eigen als Heinrich Heine, der in seiner Abhandlung „Zur Geschichte der Religion und Philosophie in Deutschland“ (von 1835) Lessings "Apostrophe an Luther" mit den scharfzüngigen Worten paraphrasierte:

Luther, du! - Großer, verkannter Mann! Und von niemanden mehr verkannt als von den Starrköpfen, die, deine Pantoffeln in der Hand, den von dir gebahnten Weg schreitend, aber gleichgültig daherschlendern! - Du hast uns von dem Joche der Tradition erlöst: wer erlöset uns von dem unerträglicheren Joche des Buchstabens! Wer bringt uns endlich ein Christentum, wie du es jetzt lehren würdest, wie es Christus selbst lelıren würde! ${ }^{23}$

In ihrer Konsequenz führte diese „Apostrophe" freilich nicht zu dem, „was Mendelssohn im Mosaismus gefunden, was die Freimaurer gesungen, was die Poeten gepfiffen “, kurz, zu dem, was der damalige Heine unter einer emanzipierten Geistigkeit verstand, sondern zu dem, was Jesus, mit der Schlußwendung der Apostrophe gesprochen, heute lehren und verkünden würde. Sie führt somit zu dem Wort, von dem Heine im Anlauf zum Lessing-Kapitel sagt, daß es, solange es lebe, von Zwergen getragen werden könne, daß es aber noch nicht einmal von Riesen aufrechterhalten werden könne, wenn es erst einmal tot sei. Das dämmerte bereits dem großen Gesprächspartner Lessings, Kierkegaard, der im Zug seiner Auseinandersetzung mit einem Christentum, das nach Lessing den Beweis des Geistes und der Kraft eingebüßt hatte, ein neues Organ für die Stimme Jesu entwickelte, so daß er selbst in den freudigsten Herrenworten den Unterton des "geheimen Leidens" vernahm. ${ }^{24}$ Fast gleichzeitig mit ihm nannte es der profilierte Vertreter der theologischen Romantik, Johann Adam Möbler, den eigentlichen Vorzug der Schrift, daß sie die spezifische „Form der Reden Jesu" enthalte; und er fügte dem das bewegende Eingeständnis hinzu, daß er nicht mehr leben möchte, wenn es ihm nicht mehr möglich sei zu hören, „wie der Sohn Gottes sprach“25.

\subsection{Frage nach der Wortverkündigung Jesu}

Es hat nicht den Anschein, als habe die heutige Theologie die ihr damit zugewiesene Aufgabe schon voll begriffen. Denn wenn in den schriftlichen Zeugnissen der lebendige Geist, paulinisch gesprochen, „eingesargt" ist und es nur die Not der Uberlieferung mit sich brachte, daß Bücher geschrieben werden mußten, kommt alles darauf an, diese wiederum, im Gegensinn zum Verschriftungsprozeß, „zum Reden zu bringen“. Was nicht zuletzt auch im Blick auf Lessings Christentumskritik gefordert ist, ist demnach ein Akt der Rückübersetzung, in dem es aber nicht um eine philosophische Reduktion, sondern darum geht, die Texte auf ihr ursprüngliches Gesprochensein zurückzuführen. ${ }^{28}$ Wenn sich das theologische Problembewußtsein dazu auch noch nicht durchzuringen vermochte, wendet sich die neueste Forschung doch mit wachsender Aufmerksamkeit der Wortverkündigung Jesu und dem daran anknüpfenden Traditionsprozeß zu. ${ }^{27}$ Danach muß in einem zweifachen Sinn von einer "Versprachlichung Jesu“ "geredet werden: einmal, sofern er seine Gottes- und Selbsterfahrung im 
Wort seiner Lehre und Verkündigung an seine Jünger weitergab; zum andern, sofern das Interesse der Augen- und Ohrenzeugen von Anfang darauf gerichtet war, zusammen mit ihrem Bild von ihm auch seine Worte und Reden an die Nachwelt zu überliefern.

Dabei unterstreicht die erste These, daß die Lebenstat des historischen Jesus wesentlich in seinem Wort bestand, während die zweite die ausschlaggebende Rolle des Rezipienten hervorhob. Doch kommt weder im ersten noch im zweiten Fall die volle Tragweite zum Vorschein, weil jeder Hinweis auf die restriktive Seite des fixierenden Mediums fehlt. Denn erst, wenn diese Erkenntnis hinzugenommen wird, zeigt sich, daß das lebendige Wort Jesu nicht nur den Anfang, sondern ebensosehr auch das Ziel der theologischen Anstrengung bilden muß, und ebenso wird dann erst klar, welche Aufgabe dem Rezipienten tatsächlich zufällt. War es ihm ursprünglich aufgegeben, die Herrenworte so zu überliefern, daß sie unverfälscht in die schriftlichen Dokumente eingebracht werden konnten, so muß er es heute als seine Aufgabe begreifen, die Zeugnisse so zu interpretieren, daß ihnen der originale "Sprachlaut" Jesu entnommen werden kann. Dies jedoch, wie dem unverzüglich hinzugefügt werden muß, nicht nach Art einer nostalgischen Rekonstruktion des historischen Redens Jesu, sondern so, wie er, mit Lessing gesprochen, sein Evangelium jetzt lehren würde.

Damit aber ergeben sich drei Schwerpunkte für den als erste Konsequenz geforderten Theorieentwurf. Es geht dabei zunächst darum, die Sprachwelt Jesus aus den schriftlichen Zeugnissen der Evangelien und der neutestamentlichen Briefe zu erheben, sodann um den Versuch, den mit dem Medium Schrift verbundenen Subtraktionseffekt auszumachen, und schließlich um die Wiedergewinnung der vollen Sprachkompetenz. Der Schwierigkeitsgrad der damit gestellten Aufgabe ergibt sich schon auf den ersten Blick aus der Zirkelhaftigkeit des angedeuteten Verfahrens, das von den schriftlichen Zeugnissen ausgehen muß, um ein Bild von der Sprechweise Jesu zu gewinnen, und zugleich die Grenzen dieser Zeugnisse durchbrechen muß, um sein Ziel zu erreichen. Zweifellos wäre der damit beschriebene Zirkel unvermeidbar, wenn nicht in Gestalt des Rezipienten ein zusätzlicher Lösungsfaktor ins Spiel käme. Indessen tritt mit ihm ein Faktor auf den Plan, der, wenn auch aus der spiegelverkehrten Optik der Rezeption, das wieder einbringt, was durch den Substraktionseffekt des Mediums verlorengegangen war. Schwierigkeiten aber sind auch in der theologisch-wissenschaftlichen Forschung niemals ein Moment der Abschreckung, sondern im Gegenteil ein zusätzlicher Anreiz, sich der durch sie gekennzeichneten Aufgabe nur um so entschiedener zu widmen.

\subsection{Das Wort als Ware}

Was nun aber die Frage nach praktischen Lösungsvorschlägen anlangt, so muß sie differenziert, im Blick auf die beiden durch die Medienverwendung geschaffenen Sprachwelten gegeben werden, auf die durch die Medien nur indirekt beeinflußte Sprachwelt der direkten Kommunikation und auf die der durch die technischen $\mathrm{Me}$ dien vermittelten Rede. Was zunächst die natürliche Sprachverwendung anlangt, so sieht sie sich auf $\mathrm{zweifache}$ Weise einem "Imitationsdruck" von seiten der Mediensprache ausgesetzt: einmal durch die mit ihr gegebene Quantitätssteigerung des Redens, zum andern durch die Modellhaftigkeit ihres Stils. Machte schon das Buch das in $\mathrm{ihm}$ reproduzierte Wort durch den Umfang seiner Verbreitung zu einem ausgesprochenen Massenartikel, so gilt das in unvorstellbar höherem Maß von dem sprachlichen „Ausstoß“ der elektronischen Medien, die das Wort nach Art eines Fließ- 
bandverfahrens reproduzieren und ihm dadurch den Charakter einer Ware aufprägen. Am deutlichsten wird das durch die mit diesem Vorgang verbundene Minimalisierung der Rezeption. Während es einige Anstrengung kostet, von einem dialogisch zugesprochenen Wort „wegzuhören", ist das bei der Aufnahme des „mediogenen“ Sprachausstoßes nahezu die Regel. Was aus dem Lautsprecher von Rundfunk- und Fernsehgeräten ertönt, ist für viele, wie die tägliche Erfahrung lehrt, kaum mehr als eine Geräuschkulisse, so daß es für sie nun umgekehrt einer eigenen Anstrengung bedarf, wenn das elektronisch vermittelte Wort auch wirklich vernommen werden soll. ${ }^{28} \mathrm{Mit}$ seinem „Warencharakter" hängt sodann auch ursächlich der „stromlinienförmige" Stil dieser Sprache zusammen. Sie wirkt, besonders im Mund von geschulten Rundfunksprechern, wohltemperiert, abgeschliffen, gefällig und glatt, ganz so, wie es dem standardisierten Erscheinungsbild - dem "design “ - von Massenwaren entspricht. In einer Zeit, die allenthalben das Profil des Individuellen im Sinn des Typisch-Gängigen abschleift, geht davon ein hoher Anreiz aus. Tatsächlich ist in der gegenwärtigen Umgangssprache, auch der gehobenen, längst schon ein Trend zur modischen Floskel zu beobachten, der zweifellos mit der angesprochenen Beeinflussung zusammenhängt. Wenn im Zug dieser Entwicklung die Mediensprache nicht schon in absehbarer Zeit zur "tonangebenden" werden soll, bedarf es hier eines Aktes sprachlicher Selbstbesinnung, der sich gleichzeitig kritisch und konzentrativ gestalten müßte. Kritisch, sofern er die Verarmung durch die Mediensprache aufzudecken hätte; aber auch konzentrativ, sofern er sich insbesondere auf die spezifisch menschlichen Sprachqualitäten beziehen müßte. ${ }^{29}$

\subsection{Das informative Wort als mediengerechte Sprachform}

Demgegenüber gilt für das durch die elektronischen Medien vermittelte Wort, so paradox dies klingt, daß es möglichst mediengerecht gestaltet werden muß. Wie langjährige Erfahrung im Bereich der religiösen Medienarbeit lehrte, führt der Versuch, Formen der dialogischen Rede oder der gottesdienstlichen Wortverkündigung auf die elektronischen Medien zu übertragen, wie immer er angelegt sein mag, letztlich zu nichts. Denn die Medien filtern aufgrund ihrer Eigenstruktur gerade die Qualitäten aus, die im Dialog oder in der Verkündigung formbestimmend sind. Dagegen ist das informative Wort, wie schon eingangs ausgeführt, optimal auf ihre Eigenstruktur abgestimmt. In die volle Konsequenz durchgezogen, läuft das auf eine erhebliche Einseitigkeit hinaus. Wie bereits angedeutet, bringt sie es mit sich, daß im Zug einer strukturgerechten Medienverwendung auf das kohortative, paränetische, erbauliche und dialogische Wort Verzicht geleistet werden muß. Was jedoch am Kanon des reichgefächerten Spektrums der natürlichen und zumal der religiösen Sprache als eminente Verarmung erscheint, stellt sich aus der Sicht der Medienästhetik als harmonischer Einklang dar. Denn wie alle Erscheinungen der technischen Welt verfügen auch die Medien über eine eigene Ästhetik. Ihr zufolge wirkt alles unschön und störend, was den technischen Strukturgesetzen widerstreitet, schön und harmonisch dagegen, was ihnen entspricht. So ist die Beschränkung auf die mediengerechte Sprachform zumindest in ästhetischer Hinsicht ein Gewinn. Wenn aber der alte scholastische Grundsatz zutrifft, daß das Schöne das leuchtende Siegel des Wahren ist, hat dieser Gewinn überdies auch Wahrheitswert. Er erklärt sich gleicherweise aus einem sprachtheoretischen Grundsatz und einem religiösen Erfahrungswert. Der Grundsatz geht davon aus, daß das Wort immer dann die höchste Effektivität erreicht, wenn es in optimaler Übereinkunft mit den sich ihm jeweils anbietenden Vermittlungswegen gesprochen wird. Auch wenn diese Wege noch so schmal sind, kommt es dann - und nur dann dazu, daß es sich, wie es ihm von seinem innersten Formgesetz her eingeschrieben ist, 
selber sagen kann. Der religiöse Erfahrungswert aber ergibt sich aus der Tatsache, daß die christliche Botschaft immer dann ihre höchste Wirkung erreichte, wenn sie nicht über die Köpfe und Dinge hinweg, sondern in so enger Angleichung an sie ausgerichtet wurde, wie es ihre Treue zu sich selbst zuließ.

\section{Anmerkungen:}

1 Benjamin, Das Kunstwerk im Zeitalter seiner technischen Reproduzierbarkeit, in: Illuminationen, Frankfurt/M. 1961, 149.

2 A. a. O., 150 f. (ohne Angabe der Fundstelle).

a A. a. O., 152.

A. a. O., 154.

s Dazu André Chastel, Die Kunst Italiens II, Darmstadt 1962, 125. Nach Gustav René Hocke gilt Arcimboldo als "Meister der totalen Metamorphosen“, auf den der für seine Zeit aufregende Satz zutrifft: "Die Kraft der Phantastik hat das Vorrecht, alles was von außen auf uns eindringt, durch die Sinne aufzunehmen, es dort zu sammeln und neu zu komponieren " (Die Welt als Labyrinth, Hamburg 1957, 46).

- Dazu der Hinweis in meiner Untersuchung ,Religiöse Sprachbarrieren. Aufbau einer Logaporetik', München 1980, 338.

7 Dazu der Abschnitt, Geist und Buchstabe‘ meines Paulusbuchs ,Der Zeugec, Graz 1981, $223-227$.

8 Dazu die Programmschrift Theodor Steinbüchels, Der Umbruch des Denkens. Die Frage nach der dhristlichen Existenz erläutert an Ferdinand Ebners Menschdeutung, Regensburg 1936.

- Ebner, Wort und Liebe, Regensburg 1935, 26.

${ }^{10}$ McLuhan, Die magischen Kanäle - ,Understanding Media', Frankfurt und Hamburg 1970.

11 Unter dem Stichwort "Die Absperrung des Universums der Rede" geht Marcuse in seiner berühmten Schrift ,Der eindimensionale Mensch' (Neuwied und Berlin 1970, 103-138) auch tatsächlich auf die Transformation der Sprache in der Massengesellschaft ein, die er durch die Heraufkunft einer geradezu ,Orwellschen' Spredhweise gekennzeidhnet sieht (107 f.).

12 Cox, Verführung des Geistes, Stuttgart 1974, 261.

13 Das entspräche durchaus dem zugleich utopischen und antiquierten Doppelcharakter der Gegenwart, die ebenso durch eine signifikante Verkürzung der Differenz von Utopie und Wirklichkeit wie durch eine Uberfrachtung mit obsolet gewordenen Lebensmodellen gekennzeichnet ist.

14 Gehlen, Die Seele im technischen Zeitalter. Sozialpsychologische Probleme in der industriellen Gesellschaft, Hamburg 1957, 44-56.

15 Dazu der Abschnitt ,Der Prothesen-Gott', meiner Schrift ,Glaubensprobleme', Augsburg $1970,48 \mathrm{ff}$.

${ }_{16}$ Dazu die wichtigen Untersuchungen von Gert Kalow, Poesie ist Nachricht. Mündliche Tradition in Vorgeschichte und Gegenwart, München/Zürich 1975; Florian Coulmas, Uberschrift, Frankfurt/M. 1981 und Walter Wimmel, Die Kultur holt uns ein. Die Bedeutung der Textualität für das geschichtliche Werden, Würzburg 1981.

17 Dazu meine Untersuchung ,Der Zeuge. Eine Paulus-Befragung', Graz 1981.

18 Dazu der Abschnitt, Geist und Buchstabe' meines Paulusbuchs, 223-227.

19 Dazu die Abhandlung von Herbert Haag, Die Buchwerdung des Wortes Gottes in der Heiligen Schrift, in: Mysterium Salutis I, Einsiedeln 1965, 289-459 (mit redaktionellen Einschüben).

20 Aurelius Augustinus, Geist und Buchstabe (De Spiritu et Littera Liber unus), Paderborn $1968,3 \mathrm{f}$.

21 Nach Gerhard Ebeling, Luther, Einführung in sein Denken, Tübingen 1981, 145.

22 Eusebius, Kirchengeschichte III, 39. 
23 Heine, Zur Geschichte der Religion und Philosophie in Deutschland, Ende des zweiten Buchs.

${ }^{24}$ Dazu der Abschnitt ,Das geheime Leiden' meines Jesusbuchs, Der Helfer', München 1973, $186-192$.

25 Möhler, Die Einheit in der Kirche, $₫ 16$.

26 Dazu mein Beitrag, Mit anderer Stimme. Predigt als Rückübersetzung;, in: Internationale katholische Zeitschrift (1982) 79-112.

27 Dazu der Beitrag von Wolfgang Feneberg, Jesus der Lehrer - Jesus der Sprechendes, in: Stimmen der Zeit 107 (1982), 857-860 (unter Bezugnahme auf Rainer Riesner, Jesus als Lehrer. Eine Untersuciung zum Ursprung der Evangelien-Ǔberlieferung, Tübingen 1981 und Paul Gerhard Müller, Der Traditionsprozeß im Neuen Testament. Kommunikationsanalytische Studien zur Versprachlichung des Jesusphänomens, Freiburg/Br. 1982).

${ }^{28}$ Das steht in einem nur scheinbaren Widerspruch zu der im Vorangehenden getroffenen Feststellung, daß dem elektronisch vermittelten Wort im Vergleich zum direkt gesprochenen eine leichtere Verständlichkeit eignet, da hier die Frage der die Rezeption überhaupt erst ermöglichenden, Vorgabe im Vordergrund steht.

29 Dazu meine (noch unveröffentlichte) Vorlesungsreihe ,Vom Sprachbild zum Sprachglück. Erwägungen zum Verhältnis von Menschsein und Spraches.

\section{SUMMARY}

The word reproduced in the electronic media relates to the spoken word like the reproduction to the original. In order to theoretically analyse this difference the concepts of analytic theory of language have to be supplemented. According to this language is the symbolic expression of men to overcome the loneliness of the individual and to create social contacts. From here the consolidating language qualities can be filtered according to their information value. With the growing media use one has to ask for the consequences especially in religious communication. Do the media give a new accent in the information of faith? How shall such a ,second-hand" channelled information be used? From here there is the question for a meaningful reaction. This should include especially a re-translation of the media transmitted word into its original expression. Also important is the adjustment of the expressions according to the specifics of the media. Their special strength most probably lies in their possibilities for teaching, information and instruction.

\section{RESUME}

Le mot reproduit par les média électroniques se comporte envers le mot prononcé, comme une reproduction envers l'original. Pour une analyse théorique de cette différence, les concepts de la théorie analytique doivent être supplétifs. Le langage est donc l'expression symbolique de l'homme pour vaincre la solitude de l'individu et pour créer des contacts sociaux. De cette perspective, les qualités consolidées du langage peuvent être filtrées selon leur valeur d'information. Avec l'utilisation croissante des média, il faut se questionner sur les conséquences, surtout pour la communication religieuse. Est-ce que les média donnent un nouvel accent dans l'information de la Foi? Et comment seront utilisées ces „expériences de seconde-main"? Finalement la question se pose d'une réaction conforme au sens que devrait inclure surtout une retraduction $d u$ mot transmis par les média dans son expression originale. L'accord entre l'expression et la structure propre des média est aussi important. Leur pouvoir spécial se trouve vraisemblablement dans leurs possibilités pour l'enseignement, l'information et l'instruction. 


\section{RESUMEN}

La palabra reproducida por los medios electrónicos se relaciona a la palabra hablada como la reproducción de un original. Con el fin de analizar teóricamente esta diferencia, los conceptos de análisis teórico del lenguaje deben ser implementados.

De acuerdo con esto, el lenguaje es la expresión simbólica del hombre para superar la soledad del individuo y para crear contactos sociales. A partir de este punto las cualidades de consolidación del lenguaje pueden ser filtrados en acuerdo a su valor informativo. Con el crecimiento del uso de media, uno debe interrogarse sobre sus consequencias, especialmente en la Comunicación Religiosa. Dán los media un nuevo acento en la información de la fé ... ? - Cómo debera ser canalizada en segunda instancia este tipo de información ...?

Esto lleva a considerar la cuestión del significado de las reacciones que provoca. Esto deberîa conprender especialmente una re-traducción de la palabra transmitida por los media hacia su expresión original.

Es también importante el ajuste de las expresiones en acuerdo a la especificidad del media. Su especial fuerza reside probablemente en sus posibilidades para la enseñanza, la información y la instrucción. 